\title{
Desafios ao desenvolvimento de um currículo inovador: a experiência da Faculdade de Medicina de Marília*
}

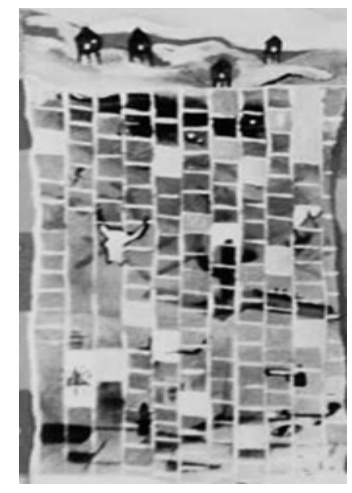

Challenges in the development of an innovative curriculum: the experience of the Marilia Medical School

\author{
Valéria Vernaschi Lima ${ }^{1}$ \\ Ricardo Shoiti Komatsu 2 \\ Roberto Queiroz Padilha ${ }^{3}$
}

\section{Introduçáo}

Por que, quando e o que mudar? Como mudar? Como avaliar a mudança? Nas últimas décadas, estas são perguntas constantes para os profissionais envolvidos com educação médica $e$ atenção à saúde em todo mundo.

Huberman (1973, p.18) considera que mudar em educação é " $a$ ruptura do hábito e da rotina, a obrigação de pensar de forma nova em coisas familiares e a de tornar a pôr em causa antigos postulados".

Esses questionamentos tomam proporções cada vez maiores quando se constata no dia-a-dia a falência dos modelos tradicionais em relação à qualidade de vida e de saúde das pessoas.

Em 1997, a Faculdade de Medicina de Marília - Famema - promoveu uma reforma curricular no curso médico e após cinco anos do desenvolvimento de um currículo centrado no estudante, baseado em problemas e orientado à comunidade, pode-se identificar algumas conquistas, mas também vários desafios a serem enfrentados nessa proposta de mudança na formação médica.

\section{Por que mudar?}

A contradição entre o crescente desenvolvimento tecnológico de um lado $e$ a baixa resolução de problemas de saúde prevalentes de outro denota a limitação das concepções tradicionais de educação médica e do modelo brasileiro de atenção à saúde.

Parte deste insucesso pode ser atribuído aos custos crescentes decorrentes da incorporação tecnológica, à política de distribuição $e$ acesso aos serviços de saúde, à redução da clínica aos aspectos biológicos $e$ à ausência ou deterioração do papel do Estado na defesa da vida e da saúde das pessoas.

${ }^{1}$ Professora, Unidade de Educação em Ciências da Saúde, Faculdade de Medicina de Marília. <valeria@famema.br>

${ }^{2}$ Professor, Unidade de Educação em Ciências da Saúde, Faculdade de Medicina de Marília. <komatsu@famema.br>

${ }^{3}$ Professor, Unidade de Educação em Ciências da Saúde, Faculdade de Medicina de Marília. <padilha@famema.br> 
O distanciamento das escolas médicas das necessidades da população, refletido pela excessiva e precoce especialização médica e tecnificação do cuidado, representa uma importante causa do inadequado perfil dos médicos formados e seu baixo compromisso social. A formação tradicional do médico transformou-se numa justaposição de disciplinas, no ciclo básico, e de especialidades, no ciclo clínico. As fragmentações básico/clínico e teórico/ prático somadas à pedagogia da transmissão representam os maiores obstáculos à aprendizagem significativa e ao desenvolvimento de um profissional crítico e capaz de trabalhar com problemas.

A necessidade de maior controle e qualidade na formação profissional e na acreditação das escolas médicas passou a estar na agenda e no foco de instituições e fóruns nacionais como o Ministério da Educação, o Ministério da Saúde, a CINAEM ${ }^{4}$ e a Rede UNIDA ${ }^{5}$.

Dessa forma, as escolas médicas brasileiras encontram-se num momento delicado, entre a necessidade de mudanças e as dificuldades de como fazê-las. Transformar a educação médica, visando uma mudança de qualidade, implica, inicialmente, uma decisão política daqueles que estão envolvidos no processo.

Na Famema a necessidade de mudanças no currículo médico vem sendo debatida desde meados da década de 1970. Criada em 1966 e mantida por uma fundação municipal, a Famema passou por sucessivas crises financeiras. A estrutura tradicional de ensino somada a uma baixa incorporação de recursos educacionais e incentivos para a qualificação docente tornava o processo ensino-aprendizagem muito mais dependente de esforços individuais. Havia entre docentes e estudantes uma insatisfação com o profissional que oferecíamos à sociedade e um desejo de inovar nas áreas de educação e saúde. Como refere Rosa (2000, p.24), toda mudança nasce da combinação entre a necessidade e o desejo e que "não há mudança sem uma certa dose de desobediência".

\section{Quando mudar?}

Cada instituição tem seus próprios tempos e movimentos resultantes da sua missão, história, organização do trabalho e características do conjunto de pessoas que dela fazem parte.

Também há regras maiores e fora do controle isolado das instituições, porém, considerando-se as pressões/influências externas à governabilidade, cada uma reage e se posiciona de modo muito particular.

Para a Famema, o Projeto UNI ${ }^{6}$, em 1992, a mudança da direção da escola, em 1993, cujo programa de gestão incluía uma proposta de reforma curricular, e a estadualização, em 1994, foram fatores que, de diferentes maneiras, catalisaram o processo e a elaboração dos novos eixos estruturantes do currículo.

A análise de quando mudar sempre será situacional, porém há que se ter um tanto de audácia, determinação e persistência porque a inércia e a tendência à norma são forças entranhadas na prática humana.

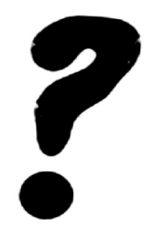

${ }^{4}$ Comissão

Interinstitucional Nacional de Avaliação do Ensino Médico

${ }^{5}$ Rede formada por instituições envolvidas nos programas UNI

(Uma nova iniciativa na formação de profissionais de saúde) e IDA (Integração docenteassistencial), ambos apoiados pela Fundação WK Kellogg.

6 Projeto UNI-Marília, iniciado em final de 1992 como parte do programa UNI da Fundação Kellogg, apoiou as reformas curriculares dos cursos de Medicina e Enfermagem da Famema. 


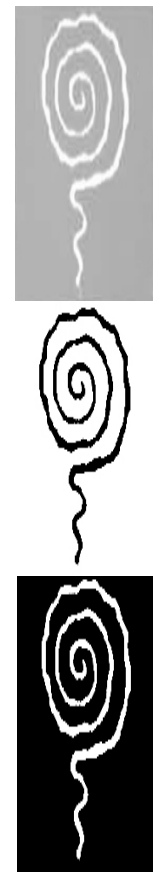

O que mudar?

O ensino tradicional está fortemente centrado no professor, que determina o que o aluno deve estudar, e em aulas magistrais, geralmente para grandes grupos. O enfoque biomédico e disciplinar somado à fragmentação entre ciclos básico/clínico, teoria/prática e trabalho/estudo limita e dificulta a aprendizagem, cabendo ao estudante integrar os diferentes saberes durante $o$ internato e na vida profissional.

Ao lidar com doentes, o médico passa a enfrentar problemas que trazem, além do componente biológico, aspectos e/ou determinantes psicológicos, econômicos, sociais e culturais. Há ainda a necessidade de aplicar o conhecimento em novas situações, integrando e articulando conteúdos disciplinares, manejando suas próprias dificuldades assim como as limitações dos pacientes e a escassez dos serviços de saúde, em busca de uma prática efetiva e humanizada.

Nessas situações, o médico necessita mais do que do conhecimento cognitivo e não há paciente que entre num consultório com uma queixa bem clara e objetiva e cinco alternativas para que se escolha a resposta mais provável.

O conhecimento, em decorrência do desenvolvimento tecnológico, vai se tornando obsoleto e para o acompanhamento dessa evolução é imperioso que o profissional de saúde tenha uma atitude contínua de aprender e habilidades para a busca e crítica das informações obtidas. A prática médica também requer destrezas psicomotoras que permitam a execução de procedimentos com segurança e técnica acurada $e$ atitudes que conformam a relação médico paciente e o trabalho em equipe. Como condutores dessa prática, sentimentos de humanidade, respeito aos direitos das pessoas $e$ compromisso social são fundamentais para o exercício profissional ético.

\section{Como mudar?}

Toda mudança requer que se explicitem os objetivos, os fundamentos e os processos do percurso. Definido o porquê mudar, buscamos referenciais que, no caso particular do Curso de Medicina da Famema, foram o currículo centrado no estudante, interdisciplinar, com aprendizagem baseada em problemas e na comunidade.

Consideramos que a chave principal do processo de mudança é entender o currículo como uma construção social que representa a totalidade das intenções e das situações de ensino-aprendizagem vivenciadas por docentes e estudantes na prática educativa. O desenvolvimento permanente do currículo significa iniciar e nunca findar, num processo contínuo de planejamento/avaliação e de reflexão sobre a coerência ou ausência de coerência entre as intenções e as ações. Nesta perspectiva, o esforço de capacitação docente torna-se essencial para alavancar mudanças $e$ desenvolver permanentemente o currículo. Só haverá transformação curricular se houver transformação da prática educacional.

Assim, programas de capacitação docente foram desenvolvidos desde o início do processo de preparação da mudança, mas intensificaram-se na fase inicial da apropriação da metodologia da aprendizagem baseada em problemas - $\mathrm{ABP}$, com a formação de tutores. Atualmente, o programa 
avançado de capacitação docente é modular, cada módulo é desenvolvido em vinte horas, $e$ os docentes selecionam dois módulos de capacitação, a cada ano letivo. São exploradas áreas tais como Bioética, Prática Baseada em Evidências, Desenvolvimento Curricular, Avaliação, Problematização, Dinâmica de Grupo, entre outras.

A construção do novo currículo da Famema, organizado em unidades educacionais, configura uma nova prática interdisciplinar. Todas as unidades educacionais são fruto de uma atuação interdisciplinar em que dez a 15 docentes em média, oriundos de diversas disciplinas e com distintas formações, desenvolvem um trabalho de construção dos objetivos da unidade, dos problemas educacionais, da organização dos recursos de aprendizagem e das atividades práticas pré-programadas. Dois dos docentes organizadores assumem a função de coordenadores do processo, $e$ as equipes de construção contam com assessoria pedagógica. Resulta desse processo a criação de unidades educacionais que não são o reflexo curricular de uma disciplina ou de uma prática unidisciplinar, mas de múltiplas disciplinas, em uma prática interdisciplinar (Komatsu et al., 2000).

A organização de recursos educacionais, especialmente os de autoaprendizagem como o Laboratório Morfo-Funcional, e o Laboratório de Informática, além da Biblioteca, também são essenciais às novas práticas de ensino-aprendizagem.

O enfoque do laboratório tradicional de ensino foi transformado para o do laboratório de (auto) aprendizagem, com organização do acervo, dos equipamentos, modelos, simuladores, peças, lâminas, para livre acesso do aluno sem a intermediação compulsória de docentes ou técnicos. O trabalho dos próprios estudantes, enquanto monitores, é fundamental ao adequado desenvolvimento das atividades nestes locais.

É importante ressaltar a relevância do processo de gestão curricular, percebendo a necessidade da criação de sistemas de controle das atividades docentes desenvolvidas nos múltiplos ambientes de ensino-aprendizagem. Nesse sentido, a Famema desenvolveu um programa de gerenciamento denominado Organização das Atividades Institucionais. Tal sistema de controle visa a otimização de recursos docentes, permitindo mapear todas as atividades docentes desenvolvidas na graduação: participação em sessões de tutoria, preceptoria de internato, ambulatórios, construção de unidades educacionais, capacitação docente etc., incluindo eventuais afastamentos para cursos ou congressos.

\section{O currículo do curso médico da Famema}

A utilização da aprendizagem baseada em problemas - ABP como estratégia de integração entre o ciclo básico e clínico, favorecendo a articulação de disciplinas e o desenvolvimento precoce do raciocínio clínico tem na Universidade de McMaster, em Hamilton, Canadá, a primeira experiência de sistematizar e organizar todo o currículo nessa abordagem, no final de 1960 (Neufeld \&

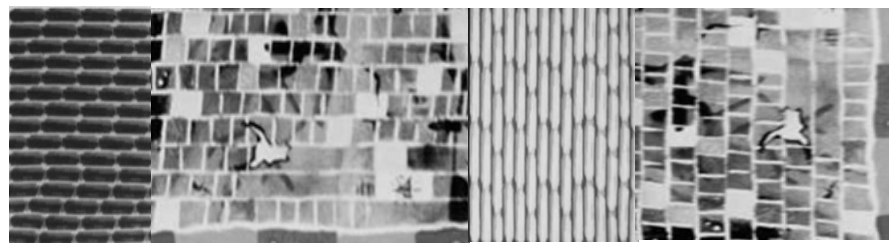
Barrows, 1974).

No decorrer das últimas décadas do século $\mathrm{XX}$, essa metodologia foi ganhando adesões de escolas 
espalhadas pelos vários continentes, chegando a casos, como o da Austrália, no qual das dez escolas médicas existentes no país, nove utilizam a ABP.

Em Marília, os passos preconizados para o desenvolvimento da ABP correspondem aos encontrados na literatura a respeito (Neufeld \& Barrows, 1974; Neame, 1981; Barrows, 1985; Schmidt, 1993; Bligh, 1995; Venturelli, 1997; Seifer, 1998). Ressaltamos, aqui, uma particularidade, no sentido de que foi proposto em Marília, para além da articulação disciplinar, uma integração das dimensões social, psicológica e biológica como uma das etapas do processamento dos problemas (Komatsu et al., 1998) e que encontra paralelo com a proposta de Campos (1999) de ampliação da clínica.

Os problemas de saúde/doença são processados nos grupos de tutoria que identificam as questões de aprendizagem a serem estudadas e discutidas na sessão seguinte. Da $1^{\text {a }}$ a $3^{\text {a }}$ séries são realizadas duas sessões semanais de tutoria além de períodos dedicados ao desenvolvimento de habilidades profissionais, quer no manejo de situações individuais (Habilidades Profissionais) ou individuais/coletivas (Interação Comunitária). Além disso, os estudantes têm períodos livres para buscar e analisar as informações necessárias de forma autodirigida.

Uma avaliação das unidades da $1^{\text {a }}$ e $4^{\text {a }}$ séries mostrou haver, na grande maioria das unidades educacionais, a presença das três dimensões já referidas. Essas, porém, aparecem de forma desarticulada. Há uma maior proporção da dimensão biológica sobre as dimensões psicológica e social (Lima, 2000); esse predomínio é esperado, uma vez que se trata de um curso da área biológica e o currículo prescrito (princípios) aponta a necessidade de integração e não de uma distribuição equivalente entre as três dimensões.

Porém, uma análise qualitativa dos objetivos educacionais e dos resultados da avaliação cognitiva apontou a existência de uma baixa qualificação das questões voltadas ao psicossocial, quando comparadas àquelas voltadas à dimensão biológica. Esta baixa qualificação se refere a uma abordagem superficializada dos aspectos psicossociais, muitas vezes focalizados pela perspectiva do senso comum. Há, entretanto, unidades educacionais nas quais a integração de dimensões, a articulação entre disciplinas básicas e clínicas e o desenvolvimento das categorias de maior hierarquia do domínio cognitivo encontram-se privilegiadas. A avaliação mostrou, ainda, que as questões de aprendizagem elaboradas pelos estudantes nas sessões de tutoria, apresentavam uma maior integração do que as questões elaboradas pelos docentes que construíram as unidades, tanto na articulação básico/clínica como entre as dimensões (Lima, 2001b).

Em relação ao desafio de integrar dimensões e articular disciplinas há fortes indicadores, obtidos pela análise dos objetivos educacionais das unidades educacionais e das questões das avaliações, de que grande parte de docentes $e$ estudantes já reconhecem a relevância e a necessidade de ampliar o modelo biomédico. Por se tratar da inclusão de novos saberes, há ainda a necessidade de melhor instrumentalizar docentes e estudantes para que essas abordagens ocorram de modo significativo e qualificado, no sentido de aumentarem a resolutividade do cuidado em saúde.

A composição da equipe de docentes que elaboram as unidades também se mostrou uma estratégia poderosa para favorecer a inclusão de conhecimentos 
das diferentes disciplinas. Quanto mais diversificada e democrática a participação de docentes das disciplinas básicas e clínicas e daqueles que são capazes de aplicar as dimensões social e psicológica na prática médica, maior a possibilidade de construção de uma unidade com uma abordagem ampliada da clínica e do modelo biomédico.

As unidades longitudinais Interação Comunitária e Habilidades Profissionais atravessam as quatro primeiras séries do curso médico e nas avaliações periódicas realizadas, como parte da avaliação do programa, tem-se tornado cada vez mais clara a necessidade de integrá-las radicalmente às unidades verticais, num movimento de unir teoria e prática, assim como, o mundo do trabalho e o do estudo.

As resistências iniciais em relação às inovações curriculares, tanto por parte de estudantes como de docentes, estão diminuindo progressivamente quer pelo melhor conhecimento da proposta educacional da Famema, por parte dos estudantes, quer pela maior experiência e domínio acumulados por parte dos docentes. A influência dos estudantes veteranos da Famema também tem sido um fator positivo na adaptação e na continência das dúvidas dos calouros.

\section{Como avaliar?}

Os elementos-chave que caracterizam um novo pensar da avaliação de programas, cursos, projetos, organizações $e$ instituições relacionam-se à finalidade e aos distintos modos de se realizar a coleta, o processamento, a análise e discussão dos resultados de uma avaliação (Worthen et al., 1997).

$\mathrm{O}$ processo avaliativo passa a ser incorporado à rotina da instituição ou programa, fazendo parte do trabalho de cada um. Todos tornam-se responsáveis pela obtenção de informação e devem perguntar-se: "que podemos fazer para melhorar o que fazemos?". Esta atitude colaborativa requer um ambiente livre de medos que permita às pessoas expressarem suas análises, evidenciando êxitos e fracassos sem temerem conseqüências.

Avaliar requer, antes de mais nada, que boas perguntas sejam elaboradas. A primeira deve ser: qual o nosso propósito ao conduzir uma avaliação? O que pretendemos avaliar? Quando avaliar? Quem irá avaliar? E, finalmente, como avaliar e para quem reportar os resultados?

Quanto maior o conhecimento dos avaliadores em relação à missão institucional e maior o domínio de instrumentos e metodologias de investigação, também maior será a precisão na elaboração de boas perguntas e procedimentos para a coleta, análise e interpretação dos dados obtidos.

Esses recursos podem ser adaptados a diferentes instituições, estimulando, assim, a auto-avaliação organizacional e a capacitação de profissionais da própria instituição para desenvolver um processo avaliativo contínuo $e$ sistemático.

\section{Avaliação de programa na Famema}

Os focos do processo avaliativo na Famema estão dirigidos para estudantes, tutores, instrutores, supervisores de estágio, profissionais de saúde, 
consultores, unidades educacionais e recursos educacionais (Lima et al., 2001a). A avaliação interna está diretamente vinculada à Direção Geral da instituição, como núcleo de assessoria técnica, sendo planejada por um grupo de docentes e representantes de estudantes dos cursos. $O$ processo avaliativo utiliza métodos quantitativos e qualitativos para coleta e análise de dados, freqüentemente de forma combinada (Lima et al., 2001a).

Os estudantes avaliam os docentes diretamente responsáveis pelo desenvolvimento de atividades educacionais em documento formal, com campos abertos e roteiro de observação. Discutem verbalmente com os docentes o resultado da avaliação e entregam um relatório, sem obrigatoriedade de identificação, na Secretaria Geral da Faculdade. A análise desses documentos é processada pelo núcleo de avaliação e enviada, junto aos documentos originais, ao núcleo de capacitação docente, que os discute com os respectivos docentes avaliados (Lima et al., 2001a).

A avaliação de todas as unidades educacionais, incluindo os recursos educacionais utilizados e/ou necessários ao desenvolvimento dessas, também é registrada formalmente em documento específico, por estudantes e docentes. O Núcleo de Avaliação prepara uma síntese dos dados coletados e os encaminha/apresenta para os responsáveis pelas unidades, coordenadores do programa e estudantes envolvidos (Lima et al., 2001a).

Os documentos originais de avaliação das unidades educacionais também são enviados aos docentes por elas responsáveis. Esses são formados por campos abertos e fechados e permitem a análise dos objetivos educacionais das unidades, dos problemas elaborados, das atividades realizadas e da organização geral da unidade (Lima et al., 2001a).

Tanto a síntese quantitativa (realizada para todas as unidades) como a qualitativa (realizada por algumas unidades escolhidas) são utilizadas para o planejamento da unidade educacional, realizado anualmente, com o objetivo de reforçar os pontos fortes identificados e superar as dificuldades ou inadequações apontadas.

Avaliação de estudantes na Famema

A avaliação formativa, voltada à melhoria do processo ensino-aprendizagem, passou a fazer parte da atividade educativa. Todos avaliam todos. Todos têm oportunidades de corrigir ou melhorar seus desempenhos.

A avaliação passou a ser dirigida aos desempenhos e não às pessoas. Fazer $e$ receber críticas passou a representar um dos desempenhos desejados, tanto para docentes como para estudantes.

A avaliação somativa, responsável pela progressão dos estudantes, está baseada em critérios representados pelos conceitos satisfatório e insatisfatório. A opção pela avaliação critério referenciada foi pautada em alguns pontoschave: estímulo para o domínio de competências; identificação de fortalezas $e$ fragilidades de cada estudante em relação a cada desempenho, orientando as atividades de aprendizagem e/ou recuperação; incentivo à colaboração ao invés da competição; controle de qualidade da certificação profissional.

Considerando-se que todos os egressos estarão automaticamente certificados para o exercício profissional da Medicina, cabe à escola garantir um padrão de qualidade para todos. 
Dessa forma, passa a importar aquilo que o estudante ainda não sabe. Passa a importar não somente a verificação do domínio cognitivo, mas também da capacidade psicomotora $e$ as atitudes do futuro profissional no cuidado à saúde das pessoas.

Na Famema, a avaliação do estudante é composta por um conjunto de instrumentos que buscam verificar em qual extensão os estudantes conseguem atingir os objetivos educacionais das unidades. Cada um dos domínios tem igual peso na progressão do estudante, sendo que alguns instrumentos, como o OSCE (Objective Structured Clinical Examination), são capazes de verificar, de forma integrada, diferentes domínios (Salvatori et al., 1995). Este tipo de avaliação é realizada por meio de estações nas quais os estudantes rodiziam. Cada estação traz, de modo simulado, diferentes situações para verificação da capacidade do estudante de identificar problemas dos pacientes (história clínica), proceder e interpretar o exame clínico e solicitar/analisar exames complementares. Muitas dessas estações utilizam pacientes simulados, porém todas as situações são estruturadas, controladas e avaliadas segundo um roteiro e padrões objetivos e previamente definidos. Esse tipo de avaliação é aplicado ao final da unidade educacional de Habilidades Clínicas da $1^{\text {a }}$ à $4^{\mathrm{a}}$ séries. Além deste, outros instrumentos compõem a avaliação somativa e autoavaliação do aluno do curso médico:

- Exercício de Avaliação Cognitiva: avaliação escrita e baseada em problemas, realizada individualmente pelos estudantes, ao final de cada unidade educacional.

- Avaliação de desempenho em atividades educacionais: avaliação da capacidade de trabalhar com problemas e em pequenos grupos, de buscar e analisar criticamente as informações e de realizar tarefas da prática profissional.

- Exercício de Investigação Científica: forma de avaliação da unidade educacional Interação Comunitária. Embora apresente uma complexidade crescente, ao longo das três primeiras séries, tem sempre por objetivo o estudo de um problema real identificado pelos estudantes na comunidade onde realizam suas atividades de aprendizagem.

- Exercício Baseado em Problema: auto-avaliação escrita e individual que reproduz as etapas da sessão de tutoria no tocante à ativação de conhecimento prévio, análise integradora dos dados do problema e levantamento de questões de aprendizagem, sendo aplicado na $1^{\mathrm{a}}$ série.

- Teste progressivo: auto-avaliação individual na forma de testes de múltipla escolha.

O estudante que obtiver um conceito insatisfatório no exercício de investigação científica, na avaliação cognitiva, no OSCE/OSPE, ou na avaliação de desempenho nas atividades educacionais (avaliações somativas) recebe um plano de recuperação específico segundo suas dificuldades e tem outras duas oportunidades nas quais é reavaliado na área de fragilidade identificada. $\mathrm{O}$ estudante deve, então, aplicar numa situação diferente os conceitos/ desempenhos considerados insatisfatórios na(s) avaliação(ções) precedente(s).

Dessa forma, o estudante somente consegue sua progressão se obtiver resultado satisfatório em todas as avaliações realizadas. Isso faz com que tanto as questões das avaliações cognitivas, como as estações das avaliações práticas sejam realmente relevantes para a aprendizagem do estudante e formação do profissional médico.

De modo geral, as avaliações têm sido bem aceitas por docentes $e$ estudantes. Quanto maior a capacidade da equipe de docentes de elaborar 
questões voltadas à alta taxonomia do domínio cognitivo, à articulação disciplinar, à integração das dimensões social, biológica e psicológica, à acurácia na realização de procedimentos, à conduta humana e ética e ao desenvolvimento do raciocínio clínico, mais próxima dos princípios curriculares estará a verificação de desempenho do estudante.

Esse processo requer um alto investimento na capacitação de docentes e na organização de suas atividades educativas, uma vez que há um consumo de tempo na construção e correção das avaliações equivalente ao empregado para a elaboração das respectivas unidades educacionais.

\section{Considerações finais}

São desafios ao desenvolvimento do currículo de Medicina da Famema, após cinco anos da implantação da reforma: sustentar as mudanças inicialmente propostas; articular efetivamente as dimensões biológica, social e psicológica, teoria e prática, educação e trabalho; prosseguir na capacitação docente permanente; continuar um processo educacional criativo e inovador.

Para isso é necessário enfrentar a ruptura de paradigmas, rompendo com práticas, processos e metodologias existentes. É relevante apontar que esse movimento implica a revisão de práticas e atitudes cotidianas e toca profundamente os valores dos educadores-profissionais de saúde.

Mudar o currículo implica mudar cada um de nós, docentes e discentes. A Famema se propõe, com entusiasmo e satisfação, a continuar na busca permanente deste "novo currículo".

\section{Referências}

BARROWS, H.S. How to design a problem-based curriculum for the preclinical years. New York: Springer Publishing, 1985.

BLIGH, J. Problem-based learning in medicine: an introduction. Postgrad Med J, n.71, p.323-6, 1995.

BLOOM, B. Taxonomía de los objetivos de la educación. Buenos Aires: El Ateneo, 1971.

CAMPOS, G. W. S. Equipes de referência e apoio matricial: um ensaio sobre a reorganização do trabalho em saúde. Cienc. Saúde Coletiva, n.4, p.393-403, 1999.

HUBERMAN, A.M. Como se realizam as mudanças em educação: subsídios para o estudo da inovação. São Paulo: Cultrix, 1973.

KOMATSU, R.S., LIMA, V.V., ZANOLLI, M.B., PADILHA, R.Q. Manual FAMEMA 1998. Marília: Faculdade de Medicina de Marília, 1998.

KOMATSU, R.S., PADILHA, R.Q., CALEMAN, G. Interdisciplinaridade na educação médica: a experiência da Faculdade de Medicina de Marília (Famema).Rev. Adm. Púb., v.6, n.34, p.81-93, 2000.

LIMA, V.V. Educação médica: a dimensão social no currículo do curso médico da Faculdade de Medicina de Marília, 1998 e 1999. São Paulo, 2000. Tese (Doutorado). Faculdade de Saúde Pública, USP.

LIMA, V.V., Pereira, S. M. S. F., ROSA, R. S. L., D’ALMEIDA, F. A., RODRIGUES, C. R., UVO, S. A. B., MOREIRA, H. M., PINHEIRO, O., FERNANDES, L. H. B., HAMAMOTO, C. G., LALUNA, M. C. C., KOMATSU, R. S., ZANOLLI, M. B., SILVA, R. F., PADILHA, R. Q., GIGLIO, E. S., MIRANDA, S. R., GUIMARÃES, A. P. C., VENTURELLI, J., BRANDA, L. A. Caderno de Avaliação. Marília: Faculdade de Medicina de Marília, 2001a.

LIMA, V.V. Comparison of tutor guide and students raised learning issues in the Nervous and 


\section{ESPAÇO ABERTO}

Endocrine units at Marilia medical School. Marília, 2001b. Dissertação (Mestrado) Health Professional Education, The University of Illinois at Chicago, UIC.

NEAME, R.L.B. How to construct a problem-based course. Med Teacher, n.3, p.94-9, 1981.

NEUFELD, V.R., BARROWS, H.S. The "McMaster philosophy": an approach to medical education. J. Med. Educ., v.49, p.1040-50, 1974.

ROSA, S.S. Construtivismo e mudança. 7.ed. São Paulo: Cortez, 2000.

SALVATORI, P., ROBERTS, J., BROWN, B. Objective Structured Clinical Examination (OSCE). In: MCMASTER UNIVERSITY. Program for educational development. Program for faculty development. Evaluation methods: a resource handbook. Hamilton: McMaster University, 1995.

SCHMIDT, H.G. Foundations of problem-based learning: some explanatory notes. Med. Educ., v.27, p.422-32, 1993.

SEIFER, S. Recent and emerging trends in undergraduate medical education. West. J. Med., n.168, p.400-11, 1998.

VENTURELLI, J. Educación médica: nuevos enfoques, metas y métodos. Washington: OPS/OMS, 1997. (Serie PALTEX Salud y Sociedad 2000, 5).

WORTHEN, B.R., SANDERS, J. R., FITZPATRICK, J. L. Program evaluation: alternative approaches and practical guidelines.2.ed. New York: Longman Publishers, 1997.

The characteristics of the process of curriculum change in the medical course of the Marilia Medical School reflect an interaction of both internal and external influences, guided by the quest for the school's greater commitment to the needs of society. The principles of the new curriculum point to a transformation of educational practices and healthcare, with a view to encouraging students to become active and critical in the building of their knowledge, but equally ethical and humane in their relations with patients/ family members and healthcare teams. The challenge of expanding clinical practice, previously limited to the biomedical model, touches upon interdisciplinary articulation, integration of the social, biological and psychological dimensions, and the combination of theory and practice and of the worlds of work and study. This challenge is faced permanently in developing the curriculum, given that this calls for changes in the practices of teachers, students and healthcare professionals.

KEY WORDS: Medical education; curriculum; program evaluation.

As características do processo de mudança curricular do curso médico da Faculdade de Medicina de Marília refletem uma interação de influências internas e externas, orientadas pela busca de um maior compromisso da faculdade com as necessidades da sociedade. Os princípios do novo currículo apontam para uma transformação nas práticas educativas e de cuidado à saúde, visando um estudante ativo e crítico na construção do seu conhecimento, bem como ético e humano na relação com pacientes/familiares e equipe de saúde. O desafio de ampliar a clínica restrita ao modelo biomédico, passa pela articulação interdisciplinar, integração das dimensões social, biológica e psicológica, união entre teoria/prática e entre os mundos do trabalho e do estudo. Esse desafio tem sido enfrentado de forma permanente no desenvolvimento curricular, uma vez que, requer mudanças na prática de docentes, estudantes e profissionais de saúde.

PALAVRAS-CHAVE: Educação médica; currículo; avaliação de programa.

Las características del proceso de cambio curricular del curso médico de la facultad de medicina de la ciudad de Marília reflejan una interacción de influencias internas y externas, orientadas por la búsqueda de un mayor compromiso de la facultad con las necesidades de la sociedad. Los principios del nuevo currículo están direccionados hacia una transformación en las prácticas educativas y de cuidado con la salud, buscando un estudiante activo y crítico en la construcción de su saber, tan ético como humano, en la relación con los pacientes/familiares y con el equipo de salud. El desafío de ampliar la clínica estricta al modelo biomédico, pasa por la articulación interdisciplinar, por la integración de las dimensiones biopsicosocial y por la unión entre teoría/práctica y entre trabajo/estudio. Ese desafío ha sido enfrentado de manera permanente en el desarrollo curricular, ya que requiere cambios en la práctica de docentes, estudiantes y profesionales de la salud.

PALABRAS CLAVE: Educación medica; currículo; evaluación de programas. 